\title{
A Linear Stability Analysis of a Homogeneous Fluidized Bed $^{1}$
}

Y.D. SOBRAL ${ }^{2}$, F.R. CUNHA ${ }^{3}$, Departamento de Engenharia Mecânica, Faculdade de Tecnologia, Universidade de Brasília, Campus Universitário Darcy Ribeiro, 70910-900 Brasília, DF, Brazil.

\begin{abstract}
In this work the local equations governing the dynamics of fluidized beds are written in terms of averaged variables and constitutive relations based on physical arguments are proposed. The averaged equations are perturbed with small disturbances from the homogeneous fluidization state, and linearized with respect to the perturbations. A stability analysis is carried out and shows that the particle pressure term has a stabilizing effect and that the particle viscosity acts as a short wave filter. The behavior of the primary instabilities described by the proposed model is in qualitative agreement with experimental observations.
\end{abstract}

\section{Introduction}

A fluidized bed is a two-phase flow system in which particles are suspended by an imposed upward fluid flow. In principle, the fluid phase could be entirely described by the Navier-Stokes equation whereas the particles could be individually described by the Newton's second law. Anderson \& Jackson [1] proposed, however, a fluidized bed modeled with particles and fluid being considered as two interpenetrating continua. The local variables describing the motion of the fluid and of the particles are replaced by smoothed variables obtained by averaging over large regions compared with the particle spacing, but small compared with the complete system. In typical problems of fluidized beds involving large particles, the relative motion of the two phases is an important part of the theory and requires the setting-up of separate equations for the average motion of the two phases [1]. This approach is different from suspensions mechanics theory that usually involves a single dynamical equation for the bulk motion, in which the effects of the particles appears in the rheological properties of the particulate system. Batchelor [2] presents a different approach for low particle Reynolds number in order to explore the physics of fluidized beds based on the hydrodynamics of particles interactions in sedimentation.

Different physical phenomena are involved in fluidization, specially at high particle Re. Anderson \& Jackson [3] were the first to develop a linear stability analysis,

\footnotetext{
${ }^{1}$ Partially supported by CNPq and FINEP-CTPetro.

${ }^{2}$ yurisobral@bol.com.br

${ }^{3}$ frcunha@unb.br
} 
who found that, in the absence of the particle pressure, fluidized beds are always unstable to any harmonic disturbance imposed to the system. In real beds, disturbances can be found in the distributor and lead to the formation of bubbles of fluid. Garg \& Pritchett [4] introduced a particle pressure term and found that fluidized beds can be stable in some conditions. There are few correlations for describing effects of particle pressure and particle viscosity in fluidized beds (e.g. $[5,6])$.

The approach of this article is to describe a fluidized bed on the phenomenological rational mechanics basis, rather than using abstract theories and hypothesis not well defined physically. Indeed, by making suitable approximations which result in tractable mathematics, our theoretical study of such particulate system has made use of constitutive relationship of physically realizable fluidized beds. As the particles interact in a fluidized bed, they exchange momentum, and this has been described here by a particle pressure. We present the continuum equations in terms of averaged properties and proceed with a linear stability analysis in order to test our model through comparison with experimental observations of primary instability, that remains one-dimensional in narrow pipes.

\section{Averaged Equations}

\subsection{Averaging Process}

The averaging process is based on a weight function defined as follows: $g(r) \rightarrow 0$ as $r \rightarrow \infty$, and $\int_{V} g(r) d V=1$, where $r$ is defined as the distance between the analyzed point $\mathbf{x}$ and an arbitrary second point, $\mathbf{y}, r=|\mathbf{x}-\mathbf{y}|$, and $V$ represents the entire volume of the system. It should be pointed out that the specific form of $g(r)$ does not need to be known, once $g(r)$ respect the above properties. The averaging process is strictly valid for $a / \ell \ll \phi^{1 / 3}$, where $\ell$ is a typical length scale of $V, a$ is a typical particle radius and $\phi$ a typical particle volume fraction.

The weight function is used to define the particle volume fraction:

$$
\phi(\mathbf{x}, t)=\int_{V_{p}(t)} g(r) d V_{y},
$$

where $V_{p}(t)$ denotes the fraction of the total volume of the system occupied by the particles at time $t$. Since $g(r)$ is a strongly decreasing function of the relative distance $r$, it will only consider the effects of the particles 'close enough' to the analyzed point. A generalized local mean property for the particulate phase, say $\bar{\Psi}(\mathbf{x}, t)$, can be defined in terms of its local point value, $\Psi(\mathbf{x}, t)$, as follows:

$$
\phi(\mathbf{x}, t) \bar{\Psi}(\mathbf{x}, t)=\int_{V_{p}(t)} \Psi(\mathbf{y}, t) g(r) d V_{y} .
$$

For a fluid phase property, this definition should be slightly modified: $\epsilon(\mathbf{x}, t)$, called the void fraction, and $V_{f}(t)$ should appear instead of $\phi(\mathbf{x}, t)$ and $V_{p}(t)$. In order to derive the averaged continuum equations, departing from the point variable continuum equations, it should be defined how to calculate derivatives of local mean 
variables. Expressions for the derivatives with respect to time and space can be derived applying the corresponding differential operator to the adequate definition of local mean property (2.2). A detailed calculation of these derivatives is available in $[7]$.

\subsection{Continuum Averaged Equations}

The averaged continuum equations are derived departing from the local point variables continuum equations for each phase. Since our interest is to describe isothermal fluidized beds of spherical particles, only the continuity and the linear momentum equations are going to be considered. Setting $\mathbf{u}$ and $\mathbf{v}$ as the fluid and the particulate local point velocities, continuity equations, considering incompressible phases, can be written as $\nabla \cdot \mathbf{u}(\mathbf{y}, t)=0$ and $\nabla \cdot \mathbf{v}(\mathbf{y}, t)=0$.

Now, setting $\rho_{f}, \mathbf{T}_{\mathbf{f}}, \rho_{p}$ and $\mathbf{T}_{\mathbf{p}}$ to denote the density and the stress tensors for the fluid and for the particulate phases, respectively, the local Cauchy's equations are written as follows:

$$
\begin{aligned}
& \frac{\partial\left[\rho_{f} \mathbf{u}(\mathbf{y}, t)\right]}{\partial t}+\nabla \cdot\left[\rho_{f} \mathbf{u}(\mathbf{y}, t) \mathbf{u}(\mathbf{y}, t)\right]=\rho_{f} \mathbf{g}+\nabla \cdot \mathbf{T}_{\mathbf{f}}(\mathbf{y}, t), \\
& \frac{\partial\left[\rho_{p} \mathbf{v}(\mathbf{y}, t)\right]}{\partial t}+\nabla \cdot\left[\rho_{p} \mathbf{v}(\mathbf{y}, t) \mathbf{v}(\mathbf{y}, t)\right]=\rho_{p} \mathbf{g}+\nabla \cdot \mathbf{T}_{\mathbf{p}}(\mathbf{y}, t) .
\end{aligned}
$$

The equations (2.3) and (2.4) are valid for all $\mathbf{y}$ in the analyzed phase. Multiplying these equations by the weight function $g(r)$, integrating in the volume occupied by the corresponding phase, and using the Reynolds' Transport Theorem in the way of our previous work [7], the average balance equations are obtained in terms of local mean properties. The continuity equations for the fluid and the particulate phases, are found to be respectively

$$
\frac{\partial \epsilon}{\partial t}+\nabla \cdot(\epsilon \overline{\mathbf{u}})=0 \quad \text { and } \quad \frac{\partial \phi}{\partial t}+\nabla \cdot(\phi \overline{\mathbf{v}})=0,
$$

and the Cauchy's equation for the particulate phase is:

$$
\frac{\partial}{\partial t}\left(\phi \rho_{p} \overline{\mathbf{v}}\right)+\nabla \cdot\left(\phi \rho_{p} \overline{\mathbf{v}} \overline{\mathbf{v}}\right)=\phi \rho_{p} \mathbf{g}+\nabla \cdot\left(\phi \overline{\mathbf{T}}_{\mathbf{p}}\right)+\sum_{N} \int_{s_{p}} g(\mathbf{x}-\mathbf{y}) \mathbf{T}_{\mathbf{p}} \cdot \mathbf{n}_{\mathbf{p}} d S_{y}
$$

Here $\mathbf{g}$ denotes the gravitational acceleration and $N$ the total number of particles in the bed. The corresponding fluid equation keeps the same form as above but $\epsilon$, $\rho_{f}, \overline{\mathbf{u}}, \mathbf{T}_{\mathbf{f}}$ and $\mathbf{n}_{\mathbf{f}}$ should appear instead of $\phi, \rho_{p}, \overline{\mathbf{v}}, \mathbf{T}_{\mathbf{p}}$ and $\mathbf{n}_{\mathbf{p}} \cdot \mathbf{T}_{p} \cdot \mathbf{n}_{\mathbf{p}}$ in the summation term of (2.6) is the traction acting on the particle-fluid interfaces. The summation over all the particles denotes the local mean force per unit of volume, $\mathbf{f}_{\mathrm{fp}}$, exerted by the fluid on the particles. In the absence of superficial stress, the Newton's $3^{\text {rd }}$ law simply implies that $\mathbf{f}_{\mathbf{f p}}=-\mathbf{f}_{\mathbf{p f}}=\mathbf{f}$.

A constitutive equation for the fluid-particle interaction force is needed. We consider here the effect of a linear drag due to the relative velocity between the phases and the effect of a transient drag (i.e. virtual mass effect) related to the 
transient acceleration of the fluid surrounding the particles. This model brings most of the physics present in a fluidized bed for arbitrary density ratios, $\chi=\rho_{f} / \rho_{p}$ and for particle Reynolds numbers $R e=\rho_{f} a u_{0} / \mu_{f}=O(1)$, with $\mu_{f}$ being the fluid viscosity and $u_{0}$ the upward mean velocity (i.e. volume flow rate of fluid, divided by the cross sectional area of the container). The parameter $\chi$ defines the type of fluidization: gas-solid (typically, $\chi \sim 0,01$ ) or liquid-solid (typically $\chi \sim 1$ ). For convenience of notation, the over-bars representing the averaged properties will be dropped. Therefore,

$$
\mathbf{f}=\alpha(\phi) \frac{\mu_{f}}{a^{2}}(\mathbf{u}-\mathbf{v})+\beta(\phi) \rho_{f}(\dot{\mathbf{u}}-\dot{\mathbf{v}}),
$$

where $\alpha(\phi)$ is defined from the Richardson \& Zaki [8] correlation function for a sedimenting sphere in a dilute dispersion and $\beta(\phi)$ is taken from the Zuber coefficient for virtual mass of a particle in an homogeneous dispersion in irrotational fluid [2]. These drag coefficients are calculated as follows:

$$
\alpha(\phi)=\frac{9}{2} \frac{\phi}{(1-\phi)^{n}}, \quad \beta(\phi)=\frac{1+2 \phi}{2(1-\phi)},
$$

where $n$ is a parameter which varies between 3 and 5 for fluidized beds [8]. The relative acceleration term $\dot{\mathbf{u}}-\dot{\mathbf{v}}$ is defined as: $\dot{\mathbf{u}}-\dot{\mathbf{v}}=\left(\frac{\partial \mathbf{u}}{\partial t}-\frac{\partial \mathbf{v}}{\partial t}\right)+(\mathbf{u} \cdot \nabla \mathbf{u}-\mathbf{v} \cdot \nabla \mathbf{v})$.

Compressible-Newtonian like stress tensors for both phases are proposed in terms of the local mean velocities and pressures. Namely

$$
\begin{gathered}
\epsilon \mathbf{T}_{\mathbf{f}}=-p \mathbf{I}+\mu_{f}\left[\nabla \mathbf{u}+\nabla^{T} \mathbf{u}-\frac{2}{3}(\nabla \cdot \mathbf{u}) \mathbf{I}\right], \\
\phi \mathbf{T}_{\mathbf{p}}=-p_{p} \mathbf{I}+\mu_{p}\left[\nabla \mathbf{v}+\nabla^{T} \mathbf{v}-\frac{2}{3}(\nabla \cdot \mathbf{v}) \mathbf{I}\right] .
\end{gathered}
$$

$\mu_{p}$ is the particulate phase viscosity, $p$ is the pressure of the fluidphase, $p_{p}$ is the particle pressure and ${ }^{T}$ denotes the transpose tensor. In this stage, $p_{p}$ and $\mu_{p}$ are considered monotone increasing function of $\phi$ only. Actually, dimensional analysis suggests that $p_{p}$ and $\mu_{p}$ may also depend on a particle temperature, defined as $\theta=\frac{1}{3} \overline{\mathbf{v}^{\prime 2}}$, where $\mathbf{v}^{\prime}$ denotes the particles mea velocity fluctuation [2]. The expressions adopted here are chosen to match an ad-hoc form for $p_{p}$ used successfully by Hernandez \& Jimenez [6], and the asymptotic expression for $\mu_{p}$ of Frankel \& Acrivos [9] derived for a statistically homogeneous suspensions in the limit of $\phi \rightarrow \phi_{c}$. Both expressions have at least some theoretical basis instead of those approximate expressions that are largely empirical: $p_{p}=\sigma G(\phi)$ and $\mu_{p}=M h(\phi)$, with

$$
G(\phi)=\phi^{3} \exp \left(\frac{r \phi}{\phi_{c}-\phi}\right), \quad h(\phi)=\frac{\phi}{1-\left(\phi / \phi_{c}\right)^{\frac{1}{3}}} .
$$

$M, r, \sigma$ are material parameters and $\phi_{c}$ is the maximum close-packing particle concentration for random suspensions. The governing equations are made nondimensional by using the scaling $u_{0}$ for velocity, $a$ for length, $\mu_{f}$ for viscosity and 
$\rho_{f} u_{0}^{2}$ for stress. The equation for the particulate phase is written as follows:

$$
\begin{aligned}
\phi\left(\frac{\partial \mathbf{v}}{\partial t}+\mathbf{v} \cdot \nabla \mathbf{v}\right)= & -\chi \sigma \nabla G(\phi)+\frac{\chi M h(\phi)}{R e}\left[\nabla^{2} \mathbf{v}+\frac{1}{3} \nabla(\nabla \cdot \mathbf{v})\right] \\
& -\phi F r \mathbf{e}_{\mathbf{3}}+\frac{\alpha(\phi) \chi}{R e}(\mathbf{u}-\mathbf{v})+\chi \beta(\phi)(\dot{\mathbf{u}}-\dot{\mathbf{v}}) .
\end{aligned}
$$

The corresponding fluid phase equation has a similar form as (2.8) and can be found in [7]. The important physical parameters of the system $F r=a \mathrm{~g} / u_{0}^{2}, \chi=\rho_{f} / \rho_{p}$ and $R e=\rho_{f} a u_{0} / \mu_{f}$ are the particle Froude number, the specific mass ratio and the particle Reynolds number, respectively. Non-dimensional continuity equations keep the same form of (2.5).

\section{Linear Stability Analysis}

The aim is now to explore by means of a normal mode analysis the dynamical behavior of a uniform fluidized bed subjected to small amplitude disturbances like a plane wave, say $\Omega=\Omega_{0} e^{\xi t} e^{i(\omega t-k z)}$. Such disturbances, usually arise close to the distributor region of real fluidized beds, are the origin of bubbles and clusters that appear in the upper region of the bed, changing completely the dynamics of the system $[10,11]$. The stable condition for this analysis is the uniform state of fluidization, one of the simplest solutions of the governing equations, $[2,3,4]: \mathbf{u}=$ $u_{0} \mathbf{e}_{\mathbf{3}}, \mathbf{v}=\mathbf{0}, \phi=\phi_{0}$ and $p=p_{0}(z)$, where $p_{0}(z)$ is the hydrostatic pressure. Small disturbances are imposed to the system in terms of non-dimensional quantities: $\mathbf{u}=\mathbf{e}_{\mathbf{3}}+\mathbf{u}_{\mathbf{1}}(z, t), \mathbf{v}=\mathbf{v}_{1}(z, t), \phi=\phi_{0}+\phi_{1}(z, t)$ and $p=p_{0}(z)+p_{1}(z, t)$.

By imposing the perturbed solution and retaining linear terms in perturbations only, the linearized governing equations of motion in terms of pare determined. The resulting system may be written as $\mathbf{A} \cdot \Omega=\mathbf{0}$, where $\mathbf{A}$ is a differential matrix operator (i.e. the modal matrix) and $\Omega$ is the vector of the amplitude of the perturbations, defined as $\Omega=\left[\begin{array}{llll}u_{1} & v_{1} & \phi_{1} & p_{1}\end{array}\right]^{T}$. Supposing plane wave perturbations of the form $\Omega=\Omega_{0} e^{s t-i k z}$, where $s=\xi+i \omega$ is the complex frequency and $k$ is the wave number of the disturbance, the resulting linearized system in the $s-k$ space is found to be: $\tilde{\mathbf{A}} \cdot \Omega_{0}=\mathbf{0}$, where $\tilde{\mathbf{A}}$ is the differential operators matrix written in the $s-k$ space. The form of $\tilde{\mathbf{A}}$ is obtained after extensive algebraic manipulations:

$$
\tilde{\mathbf{A}}=\left(\begin{array}{cccc}
-\left(1-\phi_{0}\right) i k & 0 & s+i k & 0 \\
0 & -i k & -s & 0 \\
\left(1-\phi_{0}\right)(-s-i k)+\frac{4 k^{2}}{3 R e}+ & -\frac{\alpha\left(\phi_{0}\right)}{R e}+\beta\left(\phi_{0}\right) s & -F r+\frac{\alpha^{\prime}\left(\phi_{0}\right)}{R e} & -i k \\
+\frac{\alpha\left(\phi_{0}\right)}{R e}-\beta\left(\phi_{0}\right)(s+i k) & & & \\
-\frac{\chi \alpha\left(\phi_{0}\right)}{R e}+\chi \beta\left(\phi_{0}\right)(s+i k) & -\phi_{0} s+\frac{4 \chi M h\left(\phi_{0}\right) k^{2}}{3 R e}+ & -\chi \sigma G^{\prime}\left(\phi_{0}\right) i k+ & 0 \\
& +\frac{\chi \alpha\left(\phi_{0}\right)}{R e}-\chi \beta\left(\phi_{0}\right) s & +F r-\frac{\chi \alpha^{\prime}\left(\phi_{0}\right)}{R e} &
\end{array}\right)
$$


The necessary condition for non-trivial solution of the resulting system is $\operatorname{det}(\tilde{\mathbf{A}})=0$. This leads to an algebraic quadratic equation in eigenvalues $s$ :

$$
Q_{1} s^{2}+\left(Q_{2}+i Q_{4}\right) s+Q_{3}+i Q_{5}=0
$$

where

$$
\begin{gathered}
Q_{1}=\frac{-\phi_{0}\left(1-\phi_{0}\right)-\chi \beta\left(\phi_{0}\right)}{\phi_{0}\left(1-\phi_{0}\right)}, \quad Q_{2}=\frac{4}{3} \frac{\chi M h\left(\phi_{0}\right)}{\phi_{0} R e} k^{2}+\frac{\chi \alpha\left(\phi_{0}\right)}{\operatorname{Re} \phi_{0}\left(1-\phi_{0}\right)}, \\
Q_{3}=-\frac{\left(1-\phi_{0}\right) \chi \sigma G^{\prime}\left(\phi_{0}\right)-\chi \beta\left(\phi_{0}\right)}{1-\phi_{0}} k^{2}, \quad Q_{4}=\frac{-2 \chi \beta\left(\phi_{0}\right)}{1-\phi_{0}} k, \\
Q_{5}=-\frac{F r R e\left(1-\phi_{0}\right)-\chi \alpha\left(\phi_{0}\right)-\chi \alpha^{\prime}\left(\phi_{0}\right)\left(1-\phi_{0}\right)}{\left(1-\phi_{0}\right) R e} k .
\end{gathered}
$$

The interest is to evaluate the behavior of the real part of $s$, called the growth rate of disturbances, $\xi$, against $k$. The imaginary part of $s$ denotes the frequency of the disturbances $\omega$. The velocity propagation $V=d z / d t$ of a disturbance is defined from the phase equation, $\omega t-k z=$ constant. Differentiating this relation gives, $V=\omega(k) / k$. The dispersion relation $s=s(k)$ resulting from our calculation gives

$$
\xi(k)=-\frac{-Q_{2} \pm \sqrt{\frac{\Gamma+R}{2}}}{2 Q_{1}}, \text { and } \omega(k)=-\frac{-Q_{4} \pm \frac{\Theta}{\Gamma+R} \sqrt{\frac{\Gamma+R}{2}}}{2 Q_{1}},
$$

where $R=\sqrt{\Gamma^{2}+\Theta^{2}}, \Gamma=Q_{2}^{2}-Q_{4}^{2}-4 Q_{1} Q_{3}$, and $\Theta=-4 Q_{1} Q_{5}+Q_{2} Q_{4}$.

Locally stable state for a specific wave number $k^{\prime}$ disturbance will be achieved when $\xi\left(k^{\prime}\right)<0$. When $\xi(k)<0, \forall k$, we say that the fluidized bed is linearly stable. On the other hand, if $\xi\left(k^{\prime}\right)>0$ for a specific $k^{\prime}$, the bed is locally unstable and if $\xi(k)>0$ for any $k$, the bed is said to be unstable.

The neutral line with respect to any physical parameter involved in the problem is obtained when we determine the wave numbers $k_{n}$ for which $\xi\left(k_{n}\right)=0$. Since our interest here is to evaluate the effect of the particle pressure, a neutral line with respect to the particle pressure coefficient should be plotted. An expression $\sigma=\sigma(k)$ for the neutral can be derived from (3.1). After a long algebraic manipulation, we have for $\sigma$ :

$$
\sigma=\frac{-8 Q_{1} Q_{5}\left(1-\phi_{0}\right)\left(Q_{2} Q_{4}+2 Q_{1} Q_{5}\right)+Q_{2}^{2}\left[3 Q_{4}^{2}\left(1-\phi_{0}\right)+16 Q_{1} k^{2} \chi \beta\left(\phi_{0}\right)\right]}{16\left(1-\phi_{0}\right) Q_{2}^{2} Q_{1} k^{2} \chi G^{\prime}\left(\phi_{0}\right)} .
$$

A similar expression can be determined for $M$ from (3.1) as well.

\section{Results}

The results show that both gas-fluidized beds and liquid-fluidized beds suffer the same primary one-dimensional instabilities of an uniform fluidized bed. Figure 1 (a) and Figure 1 (b) show that the growth rate of disturbances in a gas-fluidized bed (i.e. small $\chi$ ) is faster, than in a liquid-fluidized bed (higher values of $\chi$ ). 

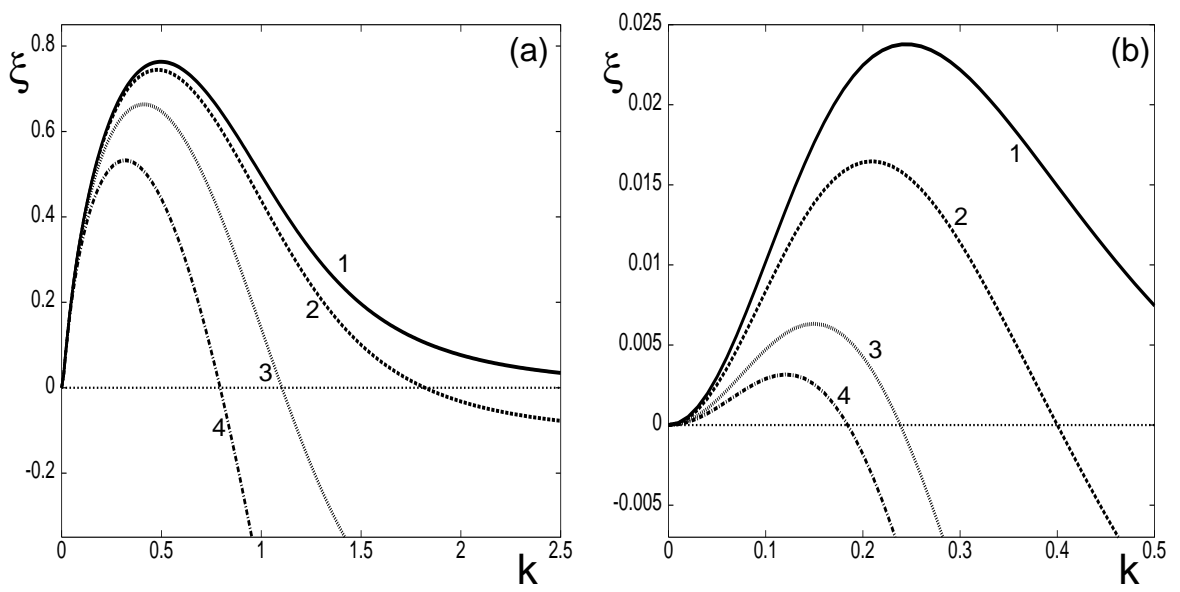

Figure 1: Growth rate of disturbances for a fluidized bed with $\operatorname{Re}=4 ; \mathrm{Fr}=10$; $n=3,65 ; \phi_{0}=0,57 ; r=0,3 ; M=50$ and $\phi_{c}=0,65$. In (a), $\chi=0,01$. The curves are identified by $1 \rightarrow \sigma=0,1 ; 2 \rightarrow \sigma=0,9 ; 3 \rightarrow \sigma=5$ and $4 \rightarrow \sigma=15$. In (b), $\chi=0,5$. The curves are identified by $1 \rightarrow \sigma=0,1 ; 2 \rightarrow \sigma=0,3 ; 3 \rightarrow \sigma=0,7$ and $4 \rightarrow \sigma=0,9$.
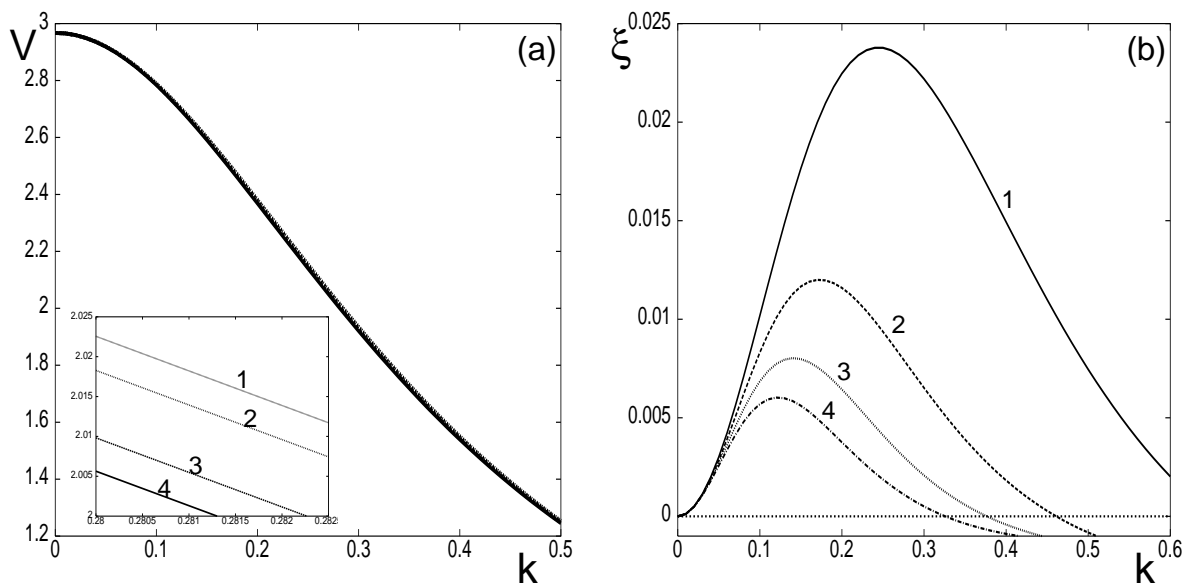

Figure 2: Results obtained for a liquid-fluidized bed with $\chi=0,5 ; \operatorname{Re}=4 ; \mathrm{Fr}=10$; $n=3,65 ; \phi_{0}=0,57 ; r=0,3 ; M=50$ and $\phi_{c}=0,65$. In (a), the velocity of propagation of disturbances is plotted. In the insert, $1 \rightarrow \sigma=0,9 ; 2 \rightarrow \sigma=0,7 ; 3$ $\rightarrow \sigma=0,3$ and $4 \rightarrow \sigma=0,1$. In (b), the effect of the particle viscosity. The curves are identified by $1 \rightarrow M=50,2 \rightarrow M=100,3 \rightarrow M=150$, and $4 \rightarrow M=200$. 

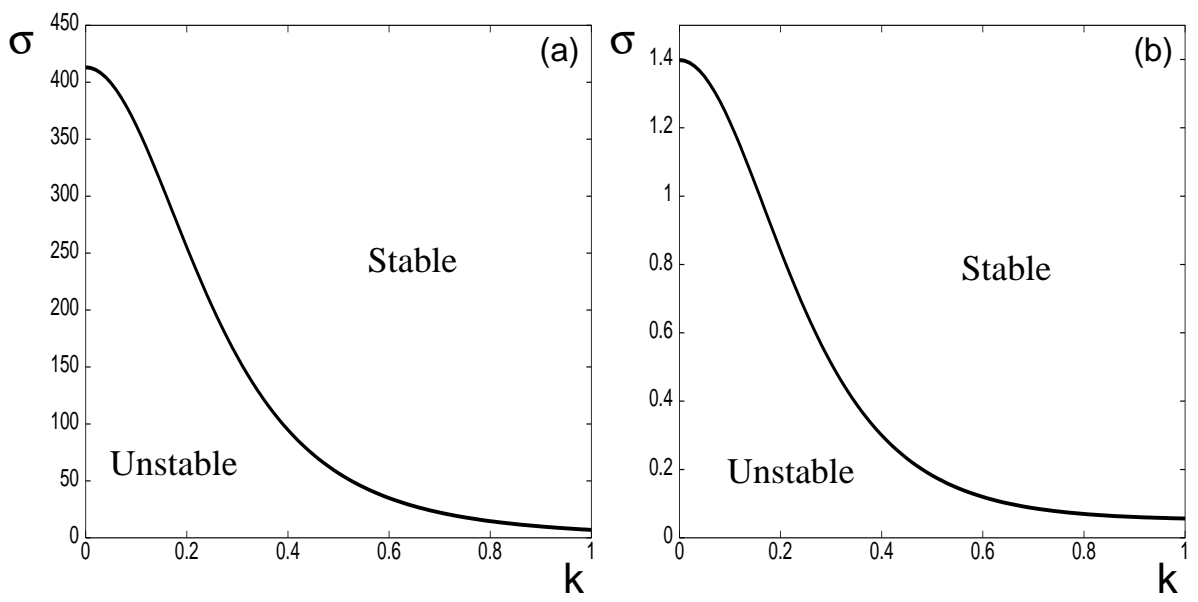

Figure 3: Neutral lines for $\sigma$ obtained for $\operatorname{Re}=4 ; \mathrm{Fr}=10 ; n=3,65 ; \phi_{0}=0,57$; $r=0,3 ; M=50$ and $\phi_{c}=0,65$. In (a), $\chi=0,01$ and in (b), $\chi=0,5$.

This difference is in general agreement with experimental observations of fluidized beds in narrow pipes [11]. It may occur because in a gas-fluidized bed the disturbances increase until a full bubble develops, whereas there is no intensification of the disturbances in a liquid-fluidized bed and then, no bubbles appear. Actually, the slower growth rate of this primary instability in liquid fluidized beds is a direct consequence of the lower fluidization velocity required. The linear theory is, however, unable to predict the mechanism of secondary instability originated by the gravitational overturning caused by a dense heavy region of the bed finding itself a light region. This is experimentally observed in wider containers [11]. The particle pressure effect has a stabilizing effect, since it reduces the region of instability for the beds, as it can be seen in Figure 1. It happens because the particles within the bed are in active motion, frequently bouncing off one another. As the particles collide, there is an exchange of momentum and this is described by the particle pressure term. A region of enhanced concentration would then be at a higher pressure and this would drive a flow to even out the disturbance. Gas-fluidized beds require a stronger particle pressure effect in order to achieve stability. On the other hand, small variations of the parameter $\sigma$ in a liquid fluidized bed caused a significant decreasing of the unstable region.

The velocity of propagation of the disturbances is plotted in Figure 2 (a) for a typical liquid-fluidized bed. The dispersive nature of the disturbances becomes clear, as it seen that different modes propagate with different velocities. This plot also shows that the changes in the velocity of the disturbances is slightly susceptible to variation of the parameter $\sigma$. The insert in Figure 2 (a) clarify this point, indicating that for $\sigma$ ranging from 0.1 to 0.9 the variations in velocity is about $1 \%$.

The particle viscosity tends to dissipate large wave number waves and their velocities tend to equalize and decay to zero as $k$ grows. In Figure $2(\mathbf{b})$, the dissi- 

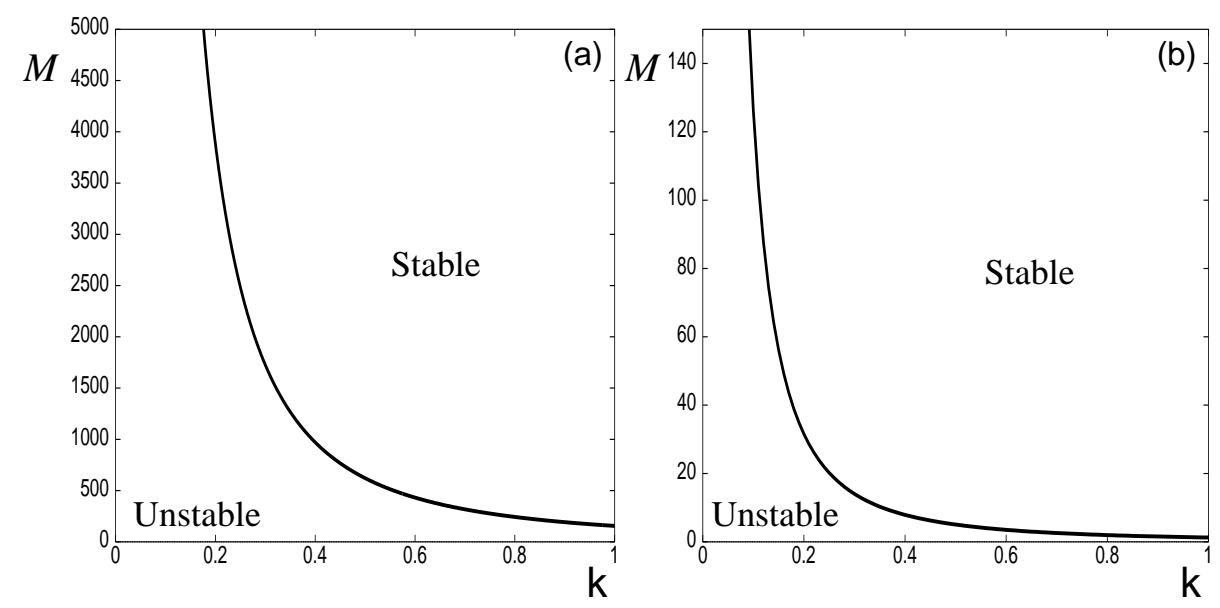

Figure 4: Neutral lines for $M$ obtained for $R e=4 ; F r=10 ; n=3,65 ; \phi_{0}=0,57$; $r=0,3 ; M=50$ and $\phi_{c}=0,65$. In (a), $\chi=0,01$ and in (b), $\chi=0,5$.

pative effect of the particle viscosity parameter is shown. Increasing the coefficient $M$ and keeping constant the other parameters, the value for critical $k$ in which a neutral growth is reached decreases. This indicates a short wave cut-off of the particle viscosity. It means that higher values of viscosity are able to dissipate modes of larger wave length. It should also be noticed that the maximum amplification rate decreases as $M$ increases, and the value of $k$ at which this happens is changed. The neutral lines in Figure 3 determine two specific regions. Stability is achieved for a specific disturbance of wave number $k$ when the particle pressure coefficient is higher than that belonging to the neutral line. It is important to note in Figure 3 (a) that the scale for $\sigma$ in gas-fluidized bed is about two orders of magnitude higher than for a liquid-fluidized bed, Figure 3 (b). The stability region dominates these plots for high values of $\sigma$, despite the wave number of the disturbances. On the other hand, particle viscosity effects lead to a stable dominant region as $k \rightarrow \infty$. Figure 4 shows that for disturbances of large wave length the region of instability dominates in both kinds of fluidization. This indicates that particle viscosity does not stabilize the fluidized bed, since there is no value of $M$ for which stability is achieved for any $k$. Thus, particle viscosity filters the modes of smallest wave length.

\section{Conclusion}

In this work, a continuum model that recovers the dynamical behavior of uniform gas and liquid fluidized beds undergoing plane wave disturbances has been proposed. The results have confirmed that liquid-fluidized beds are more stable than gasfluidized beds. The growth rates of disturbances were much smaller for the case of liquid beds and much lower values of particle pressure were required to reach stability. It was also shown that particle viscosity acts as a short wave cut-off. The 
linear stability analysis developed has predicted new analytical expressions for the growth rate, for the propagation velocity of the disturbances, and for the neutral lines of the physical parameters governing the fluidization process. The proposed model has captured the mechanism of primary instabilities of a fluidized bed, in qualitative agreement with experimental observations in narrow pipes. This primary instability has a mechanism that intensifies the growth rate of the disturbances associated with density ratio of the bed and it is seen to have a strong dispersive property. We plan to address the problem of stability of fluidized beds through a hierarchy of wave interaction analysis (KdV equations) in our future work.

\section{References}

[1] T.B. Anderson, R. Jackson, A fluid mechanical description of fluidized beds: Equations of motion, IEEEC Fundamentals, 6, No. 4 (1967), 527-539.

[2] G.K. Batchelor, A new theory of the instability of a uniform fluidized bed, Journal of Fluid Mechanics, 193 (1988), 75-110.

[3] T.B. Anderson, R. Jackson, A fluid mechanical description of fluidized beds: Stability of the uniform state of fluidization, IEEEC Fundamentals, 7, No. 1 (1968), 12-21.

[4] S.K. Garg, J.W. Pritchett, Dynamics of Gas-Fluidized Beds, Journal of Applied Physics, 46, No. 10 (1975), 4493-4500.

[5] S.E. Harris, D.G. Crighton, Solitons, solitary waves, and voidage disturbances in gas-fluidized beds, Journal of Fluid Mechanics, 266 (1994), 243-276.

[6] J.A. Hernandez, J. Jimenez, Bubble formation in dense fluidized beds, in "Proc. NATO Advanced Research Workshop on the Global Gerentry of Turbulence" (J. Jimenez, ed.), pp. 133-142, 1991.

[7] Y.D. Sobral, F.R. Cunha, A note on the governing equations of fluidized bed suspensions, em "XVI Congresso Brasileiro de Engenharia Mecânica", vol. 8, 500-509, 2001.

[8] J.F. Richardson, W.N. Zaki, Sedimentation and fluidization, Trans. Inst. Chem. Engrs., 32 (1954), 35-52.

[9] N.A. Frankel, A. Acrivos, Suspensions of non colloidal particles, in "Mobile Particulate Systems", NATO ASI Series, vol. 287, 57-68, 1995.

[10] K. Anderson, S. Sundaresan, R. Jackson, Instabilities and the formation of bubbles in fluidized beds, Journal of Fluid Mechanics, 303 (1995), 327-366.

[11] G.M. Homsy, M.M. El-Kaissy, A. Didwania, Instability waves and the formation of bubbles in fluidized beds - II, Int. Journal Multiphase Flow, 6 (1980), 305-318. 